\title{
Non Surgical-Antimicrobial Treatment of Case Type II and Case Type III Chronic Adult Periodontitis
}

\author{
Peter F.Fedi, D.D.S., M.S. \\ University of Missouri-Kansas City School of Dentistry \\ Rinehart Professor of Dentistry and chairman \\ Department of Periodontics
}

A five phase non surgical-antimicrobial program has been developed for treating early (Case Type II) and moderate (Case Type III) chronic adult periodontitis.

The non surgical-antimicrobial program consists of the following phases:

1. Case analysis.

2. Patient education.

3. Infection control.

a. Professional treatment.

b. Personal treatment.

4. Re-evaluation.

5. Maintenance.

In the short time allowed for this presentation. The antimicrobial portion of Phase 3 will be emphasized.

The goals for this form of therapy are two fold:

1. To reduce the critical mass of bacteria and their by-products to a level compatible with periodontal health.

2. To maintain this level of health for an extended period of time (measured in years).

Many studies have shown that the complete removal of subgingival plaque and calculus is impossible utilizing the instrumentation that is available today. Yet, every effort must be made to eliminate as much of this critical mass as possible and supplement this therapy with antimicrobial applications to further enhance the reduction of the critical mass of bacteria and their noxious by-products. Therefore, the Infection Control Phase consists of two steps :

1. Professional intervention, in the dental office by the doctor.

2. Personal interventions, at, home, by the patient.

Professional intervention involves the removal of root accretions by scaling, the removal of the toxic layer of cementum by root planing, polishing the root surface and the subgingival application of antimicrobials that will enhance the further reduction of the bacterial mass and their by-products.

Antimicrobials can be categorized as first generation or second generation antimicrobials.First generation antimicrobials do not have substantivity, while second generation antimicrobials exhibit this very important property.

This presentation will cover the various antimicrobials that are currently being used and methods of delivery of these in the treatment of Case Type II and Case Type III chronic adult periodontitis. 